\title{
New and Renewed Variations on Prime Numbers
}

\author{
Andri Lopez \\ Institute Polytecnic of Leon, Spain
}

Copyright (C) 2016 by authors, all rights reserved. Authors agree that this article remains permanently open access under the terms of the Creative Commons Attribution License 4.0 International License

\begin{abstract}
In this article the why and how of the prime numbers were shown; to be more specific, I present the pattern that is defined, i.e. every prime number is in the interval between $(30 a+(p))$ and $\left(42 a+\left(p_{1}\right)\right) ; \mathrm{p}=(11 ; 17 ; 23 ; 29)$; $p_{1}=(13 ; 19 ; 25 ; 31 ; 37 ; 43)$. This verifies the accuracy of the series of Dirichelet, and improvement, because any series that of a prime number matches the prime number of this pattern. Another contribution of this work is to know whether a number is prime; both for a small number, as for one he is infinitely large, without applying the process of factorization.
\end{abstract}

Keywords Arithmetic, Group $\left(G_{5} ; G_{7}\right)$, Equation Twin Prime Number

Mathematic Subject Classification $11 \mathrm{Axx}$

\section{Introduction}

If by definition a prime number is any entire number whose only divisors are unit and himself.

$$
\begin{gathered}
p \in(2 c+1) \\
\exists p \in(2 c)
\end{gathered}
$$

[1]We know that the process for have odd numbers in sum is $(1+2+2+2+\ldots .$.$) , consequently this is the same thing that$ $(3+2+2+2+\ldots .$.$) is to say (3+2 n)$. Of general form we will say $(x+x n=2 c+1)$. The prime numbers are necessary to cover the gaps left by correlative multiplication. If we multiply $3(2 c+1)$ we have the larger spaces without numbers; at multiply by the rest of the odd numbers it is obvious that the free spaces are reduced. The next number is $5=(3+2)$.

In $(3+2)(2 c+1)$ we will have the same values that we have with the number three, more $2(2 c+1)$, the value $2(2 c+1)$ fills the gaps of the number three. This is true for each and every one the products greater than three. For certain values of $[2(2 c+1)=6 n]$ we note that this value matches in the space of a product of the number three; always that $(2 c+1)=3 n$.

This synthesis shows that the primes will have a pattern that generates primes, with a difference maxim between them (consecutive) of six units.

It also verifies that:

$$
\forall\left[(30 a+(p)) ;\left(42 a+\left(p_{1}\right)\right]=2 c+1\right.
$$

because: $(p)-1=2 n$.

but not:

$$
\forall 2 c+1=\left([30 a+(p)] ;\left[42 a+\left(p_{1}\right)\right]\right)
$$

as shown by the following equation:

$$
\frac{\exists(2 c+1)-(5 ; 7)}{6} \neq Z
$$




\section{Main results}

In next step is define the interval of primes and, the because its roots are not all prime.

I begin with the series Dirichelet[2] whose numerical values are closest to zero.

$$
3+6 a ; 5+6 a ; 7+6 a
$$

The expression $(3+6 a)$ is discarded because it is always $3(2 c+1)$. The other two with a values of $(a \neq 5 n ; 7 n)$ they can not have factor and therefore we can have primes. However when we give values to (a) one of they will be a multiple of $(5 n ; 7 n)$ to avoid this multiple, we situate in (a) the following. $[5 a+(1 ; 2 ; 3 ; 4)]$ and $[7 a+(1 ; 2 ; 3 ; 4 ; 5 ; 6)]$ and we have.[1][2]

$$
\begin{gathered}
G_{5} \rightarrow 5+6[5 a+(1 ; 2 ; 3 ; 4)]=p \\
G_{7} \rightarrow 7+6[7 a+(1 ; 2 ; 3 ; 4 ; 5 ; 6)]=p
\end{gathered}
$$

it is true that we have avoided the factor $(5 n ; 7 n)$ and therefore a priori could say that all its roots are prime numbers. In its development I verified that it is not, because when (a) has the value of a product of the addends ( $\mathrm{p})$ or $\left(p_{1}\right)$, the equations they have by integer root and is not a prime.

$$
\begin{gathered}
5+6[5 a+(1 ; 2 ; 3 ; 4)]=30 a+(11 ; 17 ; 23 ; 29) \\
7+6[7 a+(1 ; 2 ; 3 ; 4 ; 5 ; 6)]=42 a+(13 ; 19 ; 25 ; 31 ; 37 ; 43)
\end{gathered}
$$

By the equations following we can know when (a) is one sum of the product (p) or $\left(p_{1}\right)$.

$$
\begin{gathered}
\frac{5+6[5 a+(1 ; 2 ; 3 ; 4)]}{(11 ; 17 ; 23 ; 29)}=Z \\
\frac{7+6[7 a+(1 ; 2 ; 3 ; 4 ; 5 ; 6)]}{(13 ; 19 ; 25 ; 31 ; 37 ; 43)}=Z
\end{gathered}
$$

To determine if an odd number is prime or not, the first one, has to belong the group $\left(G_{5} ; G_{7}\right)$.

If the roots is not an integer, then it will be a prime number, if not, it will be a product of prime number between group five and seven or among the same group in all possible combinations. Are large but finite.

In all them are true that:

$$
\begin{gathered}
\forall[30 a+(p)] \times\left[42 a+\left(p_{1}\right)\right]=\left[42 b+\left(p_{1}\right) ; 30 a+(p)\right] \\
{[3 o a+p] \times[30 a+(p)]=} \\
\left.\left[42 a+p_{1}\right] \times 42 a+\left(p_{1}\right)\right]= \\
{[3 a+(p)]^{n}=}
\end{gathered}
$$

In all these equations there is an addend that is the product of primes $(\mathrm{p})$ or $\left(p_{1}\right)$

By example: $[30 a+(p)] \times\left[42 a+\left(p_{1}\right)\right]$

(i)

$$
\begin{aligned}
& 30 a \times 42 a+30 a \times\left(p_{1}\right)+(p) \times 42 a+(p) \times\left(p_{1}\right)=\left[42 b+\left(p_{1}\right)\right] ;[30 b+(p)] \\
& 30 a \times 42 a+30 a \times(p)+\left(p_{1}\right) \times 42 a+(p) \times\left(p_{1}\right)-\left[(p) ;\left(p_{1}\right]=(42 b ; 30 b)\right.
\end{aligned}
$$

By the addend $\left(p \times p_{1}\right)$ is corroborate the why in all product of the group $\left(G_{5}\right.$ and $\left(G_{7}\right)$ we have the root of a group $\left(G_{5}\right.$ or $\left(G_{7}\right.$. As we see now.

$$
\left[(p) \times\left(p_{1}\right)\right]-\left((p) ;\left(p_{1}\right)\right)=3 n
$$

It is the only value that allows the exact division; therefore we dividing by three in (i)we have.

$\left.10 a \times 14 a+10 a \times\left(p_{1}\right)+(p) \times 14 a+n=14 b ; 10 b\right) ;(\mathrm{n})$ it is always a even number, therefore is simplified in the shape.

$$
5 a \times 7 a+5 a \times\left(p_{1}\right)+(p) \times 7 a+n^{\prime}=(5 b ; 7 b)
$$

If we substitute the value of $(5 b ; 7 b)$ in $(5 a ; 7 a)$ of the equations $5+6[5 a+(1 ; 2 ; 3 ; 4)] ; 7+6[7 a+(1 ; 2 ; 3 ; 4 ; 5 ; 6)]$.

We have a number that is origin of they and is not prime, so it is a product of two primes, however we have more options in $\left(G_{5} ; G_{7}\right)$ for define primes numbers; simply changing in the root the value of $\left(p ; p_{1}\right)$. We know the existence of nine different roots for each (a). The same is applies to each of the products and power.

Also the fundamental theorem of arithmetic it is corrobora, because if will exist a number in $\left(G_{5}\right.$ or $\left.G_{7}\right)$ that will not be prime or product of primes, then the theorem will be false. 


\section{proof.2}

Axiom: $\forall\left((p) \times\left(p_{1}\right)\right)-\left[(p) ;\left(p_{1}\right)\right]=3 n ; 5 n ; 7 m$; with $(n=2 n)$ and therefore:

$$
(p) \times\left(p_{1}\right) \notin[5+6[5 a+(1 ; 2 ; 3 ; 4)] ; 7+6[7 a+(1 ; 2 ; 3 ; 4 ; 5 ; 6]]
$$

and so is verified for being:

$$
(p) \times\left(p_{1}\right)=\left[30 a+(p) ; 42 a+\left(p_{1}\right)\right]-\left[42 a \times\left(p_{1}\right)+30 a \times(p)+42 a \times 30 a\right]
$$

With the axiom, we say that:

$$
\frac{\left[(p) \times\left(p_{1}\right)\right]^{n}-\left((p) ;\left(p_{1}\right)\right)}{3}=m
$$

This implies an equality with the product, ie.

$$
\frac{\left[(p) \times\left(p_{1}\right)\right]^{n}-\left((p) ;\left(p_{1}\right)\right)}{m}=\frac{\left.\left[(p) \times\left(p_{1}\right)\right]-\left((p) ;\left(p_{1}\right)\right)\right]}{n^{\prime}}
$$

If we subtract in they the value of three we have.

$$
\frac{\left[(p) \times\left(p_{1}\right)\right]^{n}-\left((p) ;\left(p_{1}\right)\right)}{m}-3=\frac{\left[(p) \times\left(p_{1}\right)\right]-\left((p) ;\left(p_{1}\right)\right)}{n^{\prime}}-3=0
$$

and therefore:

$$
\left[(p) \times\left(p_{1}\right)\right]^{n}-\left((p) ;\left(p_{1}\right)\right)-3 m=\left[(p) \times\left(p_{1}\right)\right]-\left((p) ;\left(p_{1}\right)\right)-3 n^{\prime}=0
$$

As admits in $\left((\mathrm{p}) ;\left(p_{1}\right)\right)$, the same value in the two terms we have:

$$
\begin{gathered}
{\left[(p) \times\left(p_{1}\right)\right]^{n}-3 m=\left[(p) \times\left(p_{1}\right)\right]-3 n^{\prime}} \\
\frac{\left[(p) \times\left(p_{1}\right)\right]^{n}-\left[(p) \times\left(p_{1}\right)\right]}{3}=m-n
\end{gathered}
$$

Whereby:

$$
\forall \frac{\left(G_{5} \times G_{7}\right)^{n}-\left[(p) \times\left(p_{1}\right)\right]}{3}=m-n^{\prime}
$$

This allows know when $(\mathrm{N})$ is one power or a product of primes Transcendent for the problem $(\mathbf{P}=\mathbf{N P})[3]$. And also is verifing that.

$$
\exists(2 c+1) \notin\left[G_{5} ; G_{7}\right]
$$

as they are:

$$
\left(3 n ; 5 n ; 7 n ;[5+6 a ; a<5] ;[7+6 a ; a<7] ;(p) \times\left(p_{1}\right) ; p \times\left(p_{1}\right) ;(p) \times(p) ; p_{1} \times\left(p_{1}\right)\right)
$$

\section{Conclusion}

Every number $\left(Z_{p}>7\right)$ has its origin in the group $\left[G_{5} ; G_{7}\right]$ and is in the interval between.

$$
[30 a+(p)] \text { and }\left[42 a+\left(p_{1}\right)\right]
$$

If this were not so, I would be inside.

$$
(2 c+1) \notin\left[G_{5} ; G_{7}\right]
$$

and no are prime.

Step to shown, as go from group five through seven, or vice versa.

$$
\begin{gathered}
G_{5} \rightarrow G_{7}=\frac{(30 a+29)+\left|2[6 a-8]-\sum 2\right|_{l m=2}}{(3 ; 5 ; 7)} \neq Z=Z_{p} \in\left(G_{5} ; G_{7}\right) \\
G_{7} \rightarrow G_{5}=\frac{(42 a+43)-\left|2[6 a+1]-\sum 2\right|_{l} m=2}{(3 ; 5 ; 7)} \neq Z=Z_{p} \in\left(G_{5} ; G_{7}\right)
\end{gathered}
$$




\section{Discussion}

First

To verify if a number $(\mathrm{N}=$ odd $)$ is prime, must meet the following equations.

$$
\begin{gathered}
\sqrt{N} \\
\frac{N}{(3 ; 5 ; 7 ; 11 ; 17 ; 23 ; 29)} \neq Z \\
\frac{N}{13 ; 19 ; 25 ; 31 ; 37 ; 43)} \neq Z \\
\frac{N-(p \times(p))}{(30 ; 42)} \neq Z \\
\frac{N-\left(p_{1} \times\left(p_{1}\right)\right)}{(30 ; 42)} \neq Z \\
\frac{N-(p) \times\left(p_{1}\right)}{(30 ; 42)} \neq Z \\
\frac{N-(p) \times\left(p_{1}\right)}{3} \neq Z
\end{gathered}
$$

The latter equation can replace the previous three.

And, for differentiate the powers and productod between primes of a prime number, also we have the following..

$$
\frac{N-5}{6}=Z \rightarrow[Z=5 a+(1 ; 2 ; 3 ; 4)]
$$

Or

$$
\frac{N-7}{6}=Z \rightarrow[Z=7 a+(1 ; 2 ; 3 ; 4 ; 5 ; 6)]
$$

Depending on which is his origin, is started the process, ie with the five or seven.

$$
\frac{Z-(1 ; 2 ; 3 ; 4)}{5}=z ; \frac{z-(1 ; 2 ; 3 ; 4)}{6}=z_{1} ; \frac{z_{1}-(1 ; 2 ; 3 ; 4)}{5} \neq z
$$

When you reach a root not exact, we pass this value $(\mathrm{z})$ to:

$$
\frac{z-(1 ; 2 ; 3 ; 4 ; 5 ; 6)}{7}=z ; \frac{z-(1 ; 2 ; 3 ; 4 ; 5 ; 6)}{7}=z ; \frac{z-(1 ; 2 ; 3 ; 4 ; 5 ; 6)}{7} \neq z
$$

If $(\mathrm{N})$ meets all the above steps; $(\mathrm{N})$ is a prime number.

\section{Second}

The last step in the development of prime number is for generate only, twin primes.

It is obvious that these are already included in $\left(G_{5} ; G_{7}\right)$ and therefore, begin has to be met the following.

$$
5 a+(1 ; 2 ; 3 ; 4)=7 a+(1 ; 2 ; 3 ; 4 ; 5 ; 6)
$$

Or from equation following.

$$
7+6[7 a+(1 ; 2 ; 3 ; 4 ; 5 ; 6)]-2=5+6[7 a+(1 ; 2 ; 3 ; 4 ; 5 ; 6)]
$$

with this equation we know, the minor of the primes, by being.

$$
\forall(5+6[7 a+(1 ; 2 ; 3 ; 4 ; 5 ; 6)]=5+6[5 a+(1 ; 2 ; 3 ; 4)])
$$

with $(a \neq a ; a>1)$ if is not met, not there are twin primes.

and if it is met, have of verify with equation following.

$$
\frac{5+6[7 a+(1 ; 2 ; 3 ; 4 ; 5 ; 6)]}{(5 ; 11 ; 17 ; 23 ; 29 ; 41)} \neq Z=p
$$

and therefore the following prime number is define with the equation following. 


$$
\frac{(p+2)}{5} \neq Z=p_{n}
$$

\section{REFERENCES}

[1] Andri Lopez; Equation for all prime number; Universal Journal of Computational Mathematic Vol 1 (3) 2013.

[2] Joan Dirichelet, on primes in arithmetic progression $a+b n, 1987$

[3] Andri Lopez; problem P=NP;in the factorization of all odd numbers; International Journal mathematic archive Vol 6 (9) 2015

[4] Bertil Nyman and Thomas R. Bien, new primes gaps betwen 1 e 15 and 5 e 16, Journal of Integer Squences 6, 2003. 